\title{
MicroRNA-130a promotes the metastasis and epithelial- mesenchymal transition of osteosarcoma by targeting PTEN
}

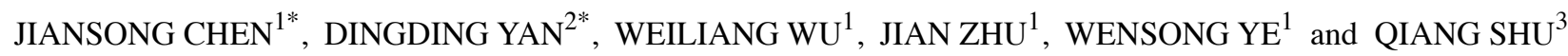 \\ ${ }^{1}$ Department of Orthopaedics, Children's Hospital of Zhejiang University, School of Medicine; \\ ${ }^{2}$ Department of Gynecologic Oncology, Zhejiang Cancer Hospital; ${ }^{3}$ Department of Tumor Surgery, \\ Children's Hospital of Zhejiang University, School of Medicine, Hangzhou, Zhejiang, P.R. China
}

Received December 17, 2015; Accepted December 30, 2015

DOI: $10.3892 / o r .2016 .4719$

\begin{abstract}
MicroRNAs, which serve as post-transcriptional modulators of numerous genes, have been found to be important regulators during the pathogenesis of osteosarcoma. This study demonstrates for the first time that microRNA-130a (miR-130a) is significantly upregulated in osteosarcoma, and associated with the metastasis of osteosarcoma. Elevated level of miR-130a was closely correlated with poor clinical features and prognosis of osteosarcoma patients. In vitro assays revealed that miR-130a could potentiate the migration, invasion and the epithelial-mesenchymal transtion (EMT) of osteosarcoma cells. Moreover, phosphatase and tensin homolog (PTEN) was confirmed as not only a direct downstream target but also a functional mediator of miR-130a.MiR-130a exerted promoting effects on metastatic behavior and EMT of osteosarcoma cells through suppressing PTEN expression. Based on these findings, we conclude that miR-130a is a promising prognostic biomarker for osteosarcoma patients, and targeting miR-130a may be a potential treatment option for osteosarcoma patients with metastasis.
\end{abstract}

\section{Introduction}

Osteosarcoma is the most common non-hematological primary bone tumor affecting children and adolescents (1), and patients with osteosarcoma have unsatisfactory prognosis, especially for those with metastasis. Currently available treatments have limited efficacy for osteosarcoma with metastasis $(2,3)$. Therefore, exploring the molecular mechanism of the metastasis of osteosarcoma is critical for identifying more effective therapeutic targets that would significantly improve the prognosis of osteosarcoma patients.

Correspondence to: Dr Qiang Shu, Department of Tumor Surgery, Children's Hospital of Zhejiang University, School of Medicine, 57 Zhugan Xiang, Hangzhou, Zhejiang 310003, P.R. China

E-mail: shu904qiang@163.com

*Contributed equally

Key words: microRNA-130a, osteosarcoma,PTEN, tumor metastasis
MicroRNAs (miRNAs) are small non-conding RNAs and act as post-transcriptional regulators of gene expression by interacting with 3'-UTR of targeted mRNAs and repressing the stability or translation of targeted mRNAs (4). Numerous studies have demonstrated that miRNAs played active roles in various cellular functions (5) including cell growth, differentiation and motility. Abnormal expression and function of miRNAs has been confirmed to play fundamental roles in human malignancies (6). In addition, miRNAs have emerged as promising biomarkers for the diagnosis and prognosis of cancer patients and attractive targets for cancer treatments (7).

MicroRNA-130a (miR-130a) has been described to participate in the development and progression of various human cancers. It was found to be overexpressed in gastric cancer $(8,9)$, esophageal cancer tissue $(10)$, and basal cell carcinoma (11), suggesting an oncogenic role in human cancer. However, studies of human hepatocellular carcinoma $(12,13)$, prostate carcinoma $(14,15)$ and breast cancer $(16,17)$ showed that miR-130a was downregulated and played tumor suppressive roles in these cancers. Therefore, the expression status and biological role of miR-130a seems to be dependent on the cancer type. However, the expression level, clinical significance and biological function of miR-130a in osteosarcoma remain unclear.

In this study, we confirmed that miR-130a expression was significantly increased in osteosarcoma tissues. Higher expression of miR-130a was observed in patients with metastasis. Elevated miR-130a expression was closely associated with poor clinicopathological features and prognosis of osteosarcoma patients. Functional assays showed that miR$130 \mathrm{a}$ promoted the migration and invasion of osteosarcoma cells by promoting epithelial mesenchymal transition (EMT). Furthermore, we identified phosphatase and tensin homolog (PTEN) as the downstream target of miR-130a in osteosarcoma cells. miR-130a exerted its functional influence on osteosarcoma cells by suppressing the expression of PTEN.

\section{Materials and methods}

Clinical specimens. Tumor specimens and the adjacent noncancer tissues were obtained from 86 osteosarcoma patients who underwent surgery in the Department of Orthopaedics, Children's Hospital of Zhejiang University School of Medicine 
between January 2003 and January 2010. Written informed consents were obtained from all participating patients. Clinical specimens were frozen and stored at $-80^{\circ} \mathrm{C}$. The patients did not receive any chemo- or radiotherapy before operation. The demographic and clinicopathological features of all included patients are presented in Table I. All protocols involving clinical samples were approved by the Ethics Review Committee of Children's Hospital of Zhejiang University School of Medicine.

Reverse transcription-quantitative PCR (RT-qPCR). Total RNA was isolated from the osteosarcoma tissues and cells using TRIzol (Life Technologies) according to the manufacturer's instructions. The TaqMan Human MiRNA Assay kit (Applied Biosystems, Foster City, CA, USA) and a SYBR ${ }^{\circledR}$ Premix Ex Taq ${ }^{\text {TM }}$ II (Perfect Real-time) kit (Takara Bio, Shiga, Japan) were employed to perform the PCR amplification. The primer of miR-130a (HmiRQP0156), PTEN (HQP015535), U6 (HmiRQP9001) and GAPDH (HQP006940) were obtained from Genecopoeia (Guangzhou, China). The relative expression of miR-130a is shown as fold difference relative to U6 while the relative expression of PTEN is shown as fold difference relative to GAPDH.

Cell culture and transfection. Human osteosarcoma cell lines HOS58, SaoS-2 and MG63, and the human normal osteoblasts (hFOB1.19) were grown in complete Dulbecco's modified Eagle's medium (DMEM; HyClone, Logan, UT, USA) supplemented with $10 \%$ fetal bovine serum (FBS; HyClone), $100 \mathrm{U} / \mathrm{ml}$ penicillin, and $100 \mu \mathrm{g} / \mathrm{ml}$ streptomycin. Cell cultures were incubated in a humidified incubator containing of $5 \%$ $\mathrm{CO}_{2}$ at $37^{\circ} \mathrm{C}$.

miR-130a expressing vector (HmiR0170-MR03), the control vector (CmiR0001-MR03), miR-130a inhibitor (HmiR-AN0156-AM03) and the negative control (CmiRAN0001-AM03), were purchased from GeneCopoeia. Plasmids carrying PTEN expressing vector (\#28298) and PTEN specific shRNA (\#25638) were obtained from Addgene (Cambridge, MA, USA). The vectors mentioned above were transfected into the osteosarcoma cells using Lipofectamine 2000 (Invitrogen, Carlsbad, CA, USA) following the manufacturer's protocol.

Transwell assays. The migration and invasion of osteosarcoma cells were evaluated in Transwell chambers with $8-\mu \mathrm{m}$ inserts (Millipore Corp., Billerica, MA, USA) according to the manufacturer's instructions. Osteosarcoma cells were resuspended in serum-free medium and seeded into the top chamber of each insert. Serum-containing medium (10\% FBS) was used in the lower chamber as the attractant. In the invasion assay, each upper chamber was coated with mixture of DMEM and Matrigel (Becton-Dickinson Labware, Bedford, MA, USA) at a ratio of 8:1, and $24 \mathrm{~h}$ after cell seeding, the cells in the upper surface of the filter were removed with a cotton swab, and the migrated or invaded cells were stained with $0.1 \%$ crystal violet. Cell numbers on the lower surface were counted. Three independent experiments were performed.

Western blotting. Cells were collected and washed twice with phosphate-buffered saline (PBS), and lysed with RIPA lysis buffer (BioMed, Beijing, China). Lysate was centrifuged at $14,000 \mathrm{rpm}$ for $20 \mathrm{~min}$ and the supernatants containing protein were collected. Protein concentration was measured using the BCA kit (Pierce, Rockford, IL, USA). Protein (30-40 $\mu \mathrm{g}$ ) of each sample was separated and transferred to PVDF membrane. The blots were incubated with the following primary antibodies: PTEN (1:1,000, Cell Signaling Technologies, Danvers, MA, USA), E-cadherin (1:1,000, Cell Signaling Technologies), vimentin (1:1,000, Cell Signaling Technologies) and GAPDH (1:1500, Santa Cruz Biotechnology, Santa Cruz, CA, USA). After washing the membranes with TBST, blots were incubated with anti-mouse or anti-rabbit secondary antibodies (1:10,000; Bio-Rad, Hercules, CA, USA), and the signals were detected using the Bio-Rad Gel imaging system.

Luciferase reporter assay. Wild-type 3'-UTR sequence of PTEN (wt PTEN-3'-UTR) which was predicted to interact with miR-130a and the mutated sequence (mt PTEN-3'-UTR) were synthesized and inserted into the pGL3 control vector (Promega, Madison, WI, USA). For luciferase reporter assay, cells were transfected with the wild-type construct or mutant construct, and miR-130a expressing vector, miR-130a inhibitor, control vector or negative control. At $48 \mathrm{~h}$ after transfection, the luciferase activity was measured using the Dual-Luciferase Reporter Assay system (Promega, Shanghai, China) with a luminometer (Promega). Results were obtained from three independent experiments performed in duplicate.

Statistical analysis. SPSS statistical package for Windows version 13 (SPSS, Inc., Chicago, IL, USA) and GraphPad Prism 5 software (GraphPad Software, Inc., USA) were used to perform the statistical analysis, including the Pearson's Chi-square test, the Spearman's rank correlation coefficient, the Student's t-test, the Kaplan-Meier plot, and the log-rank test or ANOVA was performed in this study. $\mathrm{P}<0.05$ was considered to indicate a statistically significant difference.

\section{Results}

miR-130a expression is increased in osteosarcoma and is associated with the metastasis of osteosarcoma. Quantitative RT-PCR assay was conducted to evaluate the expression level of miR-130a in osteosarcoma tissues and matched non-tumor tissues. miR-130a in osteosarcoma tissues was increased significantly compared with that in the adjacent non-tumor tissues $(\mathrm{P}<0.05$, Fig. 1A). Moreover, in tissues with metastasis, the level of miR-130a was significantly higher than that in those without metastasis $(\mathrm{P}<0.05$, Fig. 1B). Furthermore, the expression level of miR-130a in osteosarcoma cell lines was measured. Compared with normal human osteoblastic cell line, hFOB1.19, osteosarcoma cell lines, including HOS58, SaoS-2 and MG63, had significantly higher level of miR-130a $(\mathrm{P}<0.05$, Fig. 1C). MG63 and SaoS -2 cells had obviously higher expression level of miR-130a than HOS58 cells $(\mathrm{P}<0.01$, Fig. 1C). These results indicate that miR-130a is significantly upregulated in osteosarcoma and is associated with the metastasis of osteosarcoma.

High expression level of miR-130a is correlated with adverse clinicopathological features and poor prognosis of osteosar- 

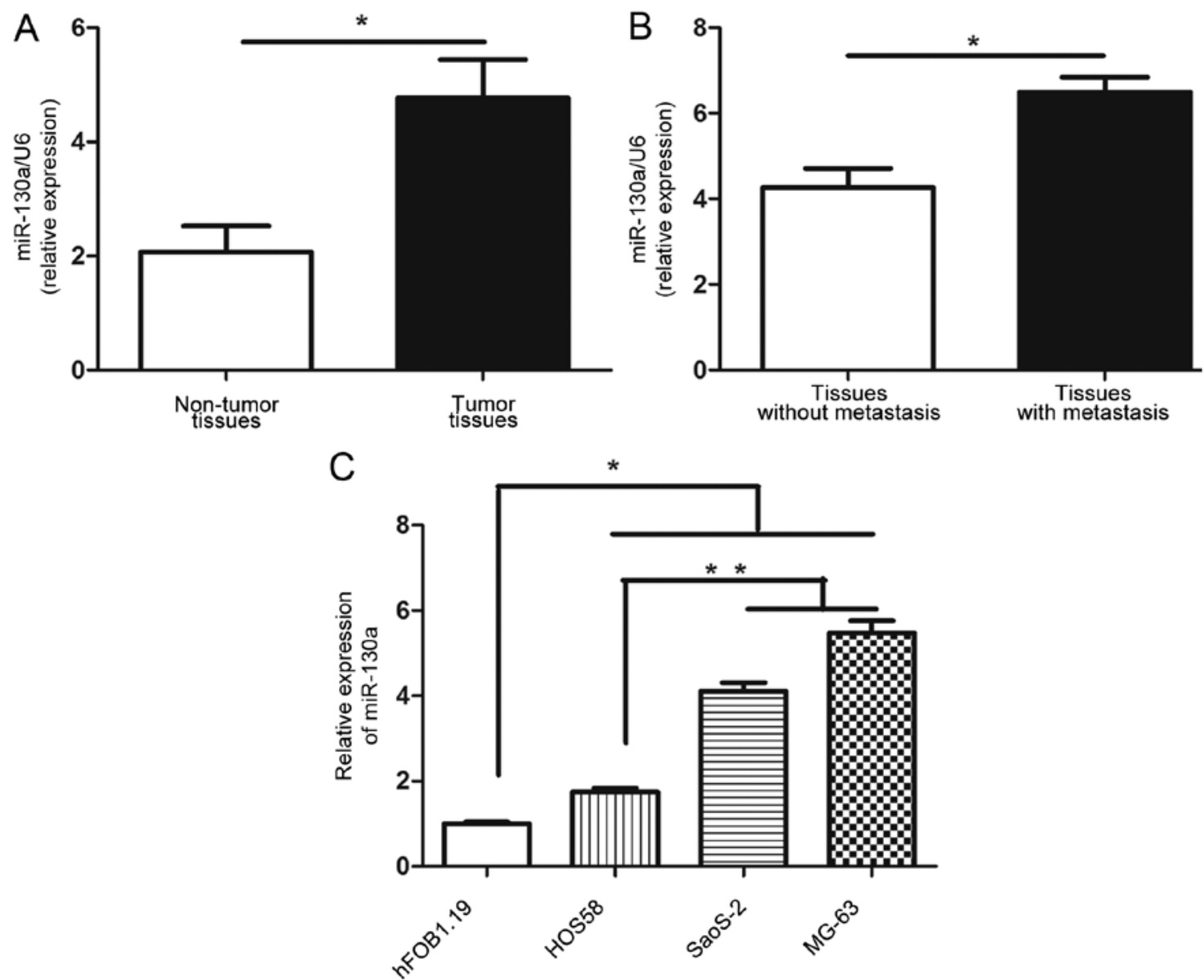

Figure 1. The expression levels of miR-130a in osteosarcoma tissues and cells. The differences of the expression levels of miR-130a between (A) osteosarcoma and adjacent non-tumor tissues, (B) tissues with metastases and those without metastases, and (C) different osteosarcoma cell lines (HOS58, SaoS-2, and MG63) and normal human osteoblastic cell line (hFOB1.19). ${ }^{*} \mathrm{P}<0.05,{ }^{* *} \mathrm{P}<0.01$.
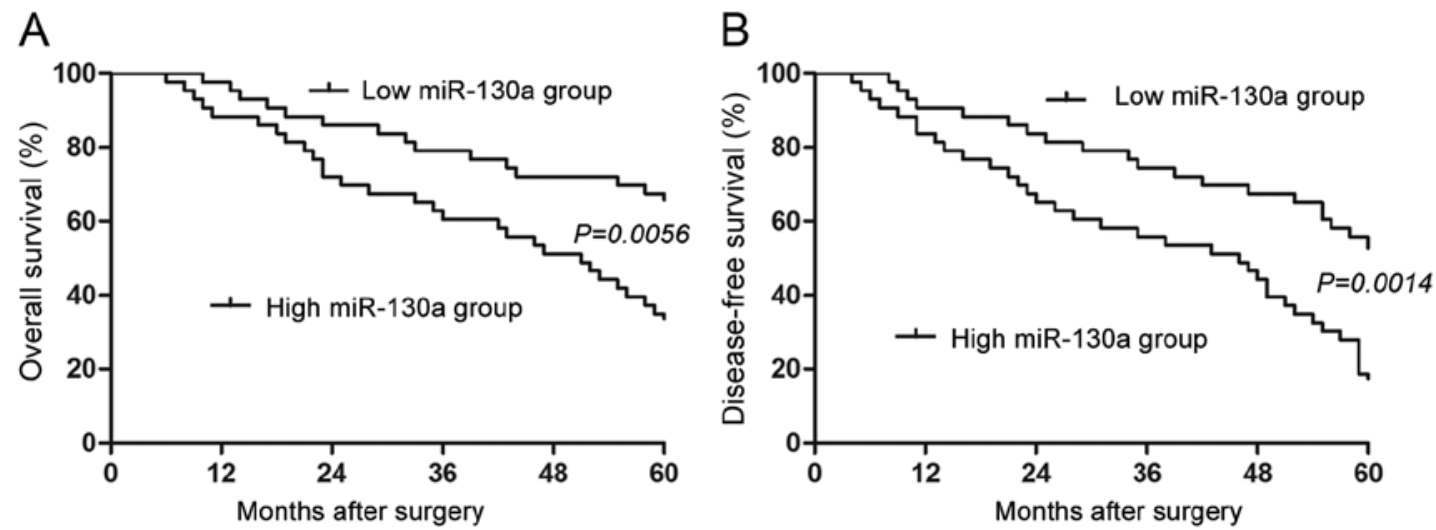

Figure 2. The prognostic value of miR-130a for osteosarcoma patients. Compared with those with low miR-130a level, patients with high miR-130a level had significantly shorter (A) overall survival (OS) and (B) disease-free survival (DFS). The median expression level of miR-130a in 86 osteosarcoma patients was chosen as the cut-off value.

coma patients. Next, the clinical significance of miR-130a expression level in osteosarcoma tissues was examined. The expression of miR-130a was assessed either low $(n=43)$ or high $(n=43)$ based on the median level of miR-130a in the 86 patients cohort. As presented in Table I, high expression level of miR-130a in osteosarcoma patients was closely associated with metastasis $(\mathrm{P}=0.002)$ and advanced TNM stage $(\mathrm{P}=0.023)$. Importantly, Kaplan-Meier analysis further showed that patients with high miR-130a expression level had significantly shorter overall survival $(\mathrm{P}=0.0056$, Fig. $2 \mathrm{~A})$ and disease-free survival ( $\mathrm{P}=0.0014$, Fig. $2 \mathrm{~B})$. These results suggest miR-130a is a promising prognostic predictor for osteosarcoma patients.

miR-130a promotes the migration, invasion and EMT of osteosarcoma cells. The functional role of miR-130a in 

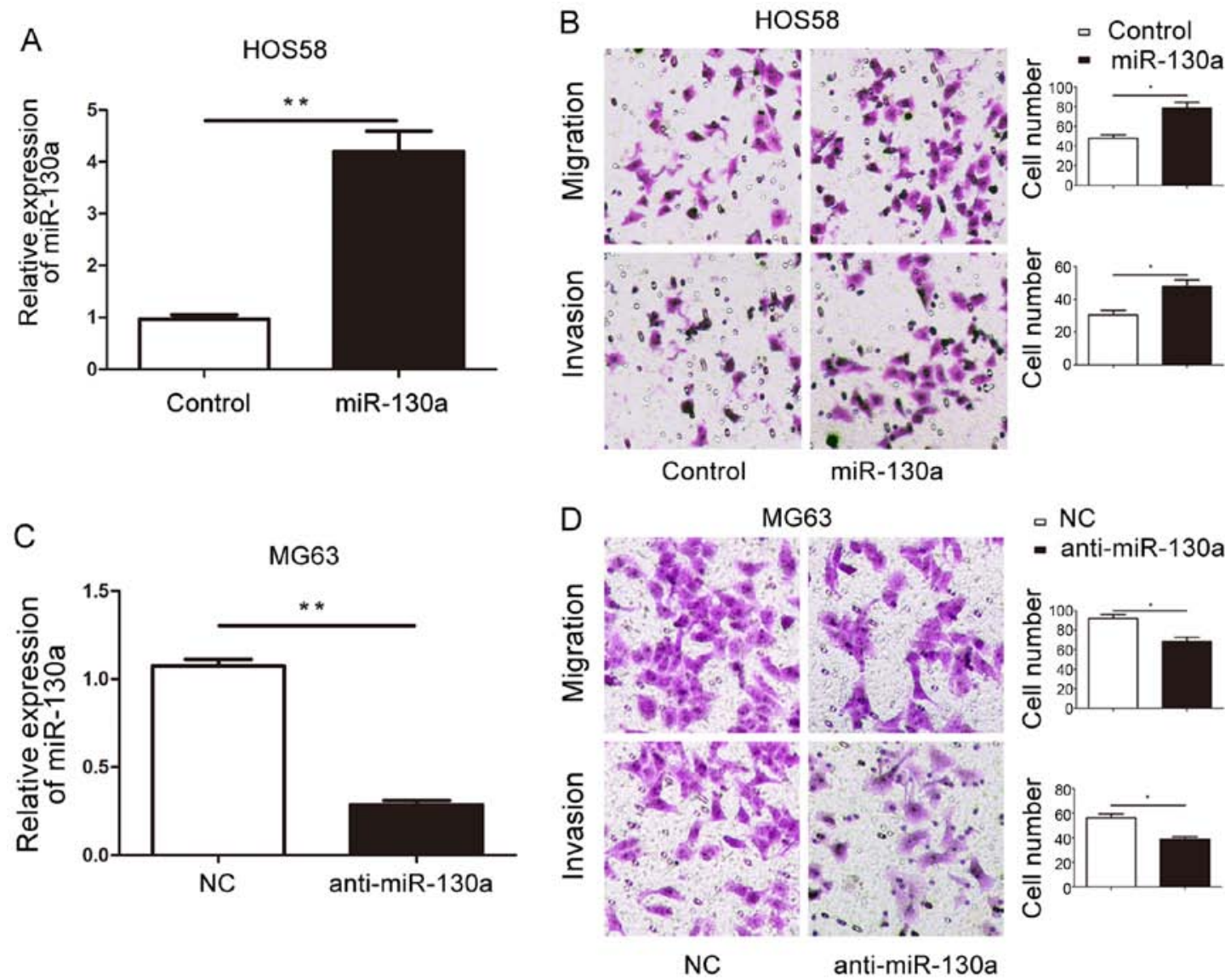

$\because \mathrm{NC}$

- anti-miR-130a
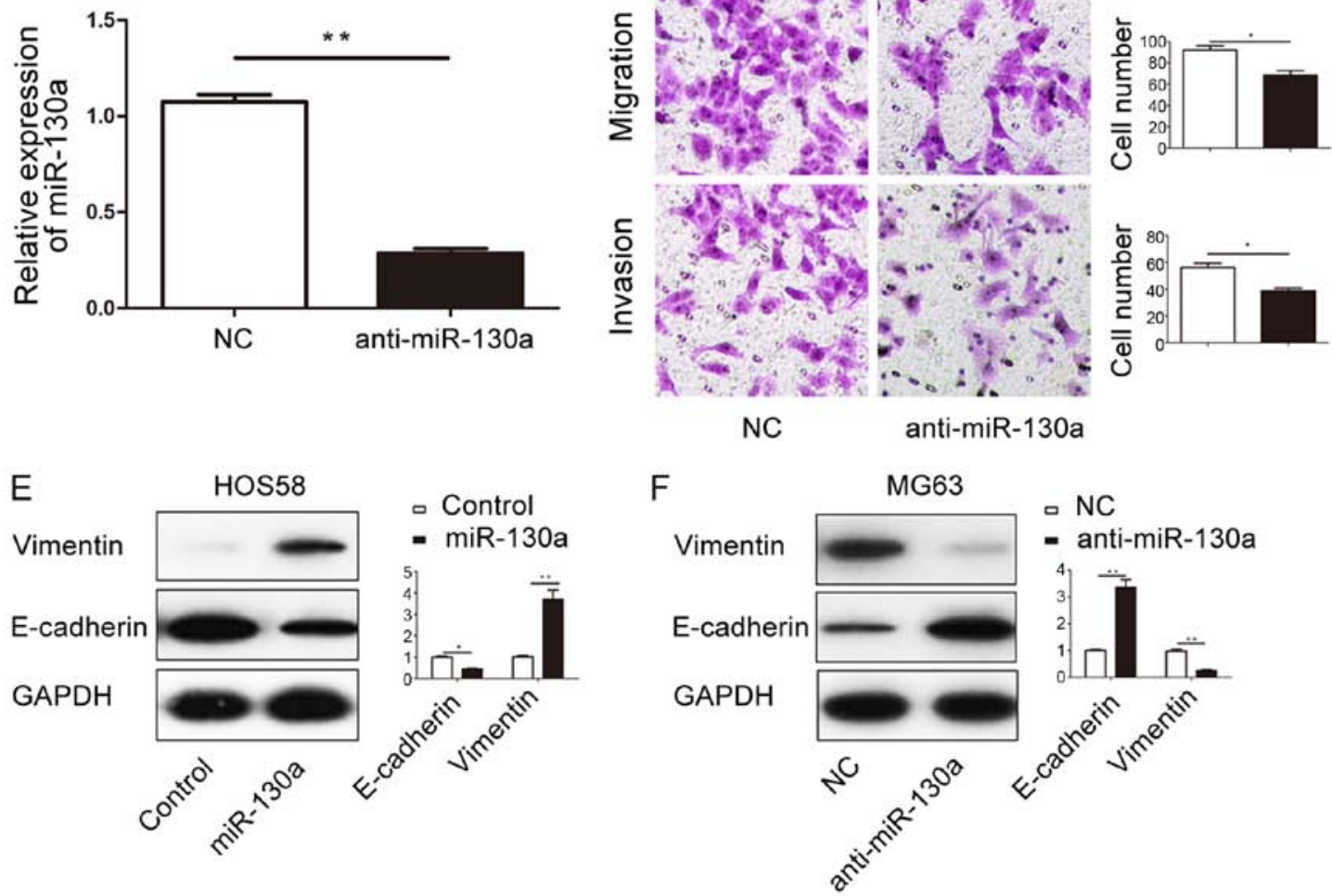

Figure 3. miR-130a promotes the migration, invasion and EMT phenotype of osteosarcoma cells. (A) miR-130a expressing vector significantly increased the level of miR-130a in HOS58 cells; ${ }^{* * *} \mathrm{P}<0.01$. (B) Overexpression of miR-130a significantly increased the migration (upper part) and invasion (lower part) of HOS58 cells. $n=3$ repeats with similar results; ${ }^{*} \mathrm{P}<0.05$. (C) miR-130a inhibitor significantly decreased the level of miR-130a in MG63 cells; ${ }^{* *} \mathrm{P}<0.01$. (D) Suppression of miR-130a significantly reduced the migration (upper part) and invasion (lower part) of MG63 cells. $\mathrm{n}=3$ repeats with similar results; ${ }^{*} \mathrm{P}<0.05$. (E) Western blot results of E-cadherin and Vimentin in HOS58 cells transfected with miR-130a mimics; $\mathrm{n}=6$; ${ }^{*} \mathrm{P}<0.05$, ${ }^{* *} \mathrm{P}<0.01$. (F) Western blot results of E-cadherin and Vimentin in MG63 cells transfected with miR-130a inhibitor; $n=6 ;{ }^{* *} \mathrm{P}<0.01$.

osteosarcoma cells was explored. HOS58 cells with miR-130a mimics, and the expression level of miR-130a was significantly increased after transfection ( $\mathrm{P}<0.01$, Fig. $3 \mathrm{~A})$. Functionally, as suggested by the Transwell assay, HOS58 cells overexpressing miR-130a (HOS58-miR-130a cells) showed increased migratory $(\mathrm{P}<0.05$, upper part in Fig. $3 \mathrm{~B})$ and invasive $(\mathrm{P}<0.05$, lower part in Fig. 3B) ability. In contrast, miR-130a inhibitor significantly downregulated the expression level of miR-130a in MG63 cells $(\mathrm{P}<0.01$, Fig. $3 \mathrm{C})$. The migration $(\mathrm{P}<0.05$, upper part in Fig. 3D) and invasion (P<0.05, lower part in Fig. 3D) of MG63 cells was significantly decreased after the expression of miR-130a was inhibited. These data indicate that miR-130a potentiate the metastatic behavior of osteosarcoma cells.
The EMT process, which has been regarded as a fundamental process of the cancer metastasis (18-20), can increase the migratory and invasive ability of osteosarcoma cells (21). Therefore, we further explored whether miR-130a promotes the migration and invasion of osteosarcoma cells by promoting EMT. The results of western blot showed that overexpression of miR-130a in HOS58 cells resulted in decreased level of E-cadherin $(\mathrm{P}<0.05$, Fig. 3E) and increased expression of Vimentin $(\mathrm{P}<0.01$, Fig. 3E). On the contrary, downregulating miR-130a in MG63 cells led to increased level of E-cadherin $(\mathrm{P}<0.01$, Fig. $3 \mathrm{~F})$ and decreased expression of Vimentin $(\mathrm{P}<0.01$, Fig. 3F). These results suggest that miR-130a can promote EMT phenotype of osteosarcoma cells. 
A

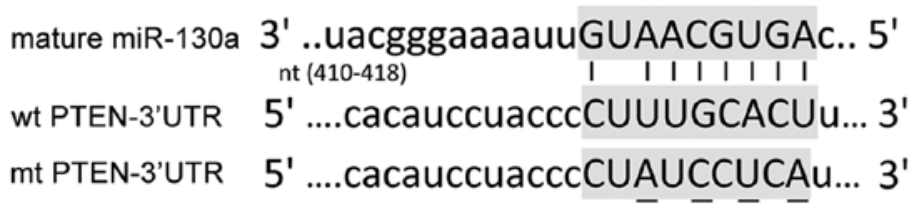

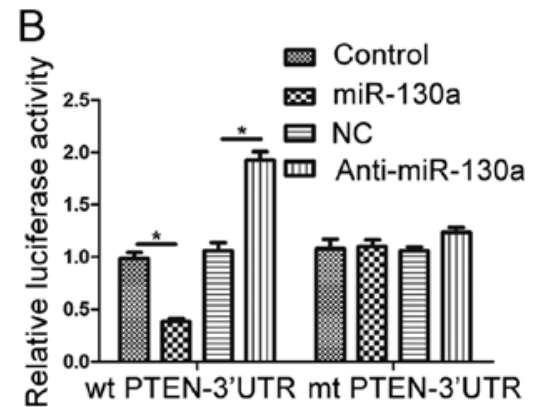

Figure 4. miR-130a interacts with the 3'-UTR of PTEN. (A) miR-130a and its predicted binding sequence in the 3'-UTR of PTEN. The mutant miR-130a binding site was generated in the complementary site for the seed region of miR-130 (wt, wild-type; mt, mutant type). (B) Alteration of the expression level of miR-130a significantly changed the luciferase activity that carried wt 3'-UTR of PTEN but had no significant influence on mt 3 '-UTR of PTEN; ${ }^{\text {P }}<0.05$.

Table I. Correlation between the clinicopathological characteristics and miR-130a expression in osteosarcoma.

\begin{tabular}{|c|c|c|c|c|}
\hline \multirow[b]{2}{*}{ Parameter } & \multirow{2}{*}{$\begin{array}{l}\text { Total no. } \\
\text { of patients } \\
\quad(n=86)\end{array}$} & \multicolumn{2}{|c|}{ No. of patients } & \multirow[b]{2}{*}{ P-value } \\
\hline & & $\operatorname{miR}-130 a^{\text {low }}$ & miR-130a high & \\
\hline Age & & & & 0.664 \\
\hline$>25$ & 49 & 22 & 27 & \\
\hline$\leq 25$ & 37 & 19 & 18 & \\
\hline Gender & & & & 0.827 \\
\hline Male & 50 & 24 & 26 & \\
\hline Female & 36 & 19 & 17 & \\
\hline Location & & & & 0.658 \\
\hline Proximal & 53 & 25 & 28 & \\
\hline Distal & 33 & 18 & 15 & \\
\hline Tumor size & & & & 0.490 \\
\hline$\leq 5 \mathrm{~cm}$ & 58 & 31 & 27 & \\
\hline$>5 \mathrm{~cm}$ & 28 & 12 & 16 & \\
\hline Differentiation & & & & 0.635 \\
\hline Well/moderate & 61 & 32 & 29 & \\
\hline Poor & 25 & 11 & 14 & \\
\hline Metastasis & & & & 0.002 \\
\hline Absent & 55 & 35 & 20 & \\
\hline Present & 31 & 8 & 23 & \\
\hline TNM stage & & & & 0.023 \\
\hline $\mathrm{I}+\mathrm{II}$ & 51 & 25 & 26 & \\
\hline III + IV & 35 & 8 & 27 & \\
\hline
\end{tabular}

PTEN is a downstream target of miR-130a in osteosarcoma cells. To further investigate the underlying mechanisms for the functional influence of miR-130a in osteosarcoma cells, two publicly available databases (TargetScan 6.2 and miRanda) were searched for the potential downstream target of miR130a. PTEN, which is a well-recognized tumor suppressor and an important participator in the development and progression of osteosarcoma, was suggested to be a potential downstream target of miR-130a. As shown in Fig. 4A, the 3'-UTR of PTEN mRNA contained the complementary sequence of miR-130a.
This suggests that miR-130a can potentially bind to the 3'-UTR of PTEN. To confirm this prediction, we performed dual-luciferase reporter assays to elucidate whether miR-130a could directly bind to the 3'-UTR of PTEN. Forced expression miR-130a significantly decreased the luciferase activity of PTEN with a wild-type (wt) 3'-UTR (P<0.01 Fig. 4B), but had no significant influence on that with a mutant (mt) 3'-UTR. On the contrary, when miR-130a inhibitor was transfected, the luciferase activity of wt PTEN 3'-UTR obviously increased $(\mathrm{P}<0.01$, Fig. 4B) while that of mt PTEN 3'-UTR remained unchanged.

Moreover, we investigated whether alteration of miR-130a could affect the expression level of PTEN mRNA and protein. qRT-PCR and western blot were performed to examine the expression level of PTEN after forced expression of miR-130a in HOS58 cells. The result of qRT-PCR showed that PTEN mRAN level in HOS58 cells was significantly decreased after overexpression of miR-130a ( $\mathrm{P}<0.01$, Fig. 5A). Consistently, the protein level of PTEN was significantly decreased after overexpression of miR-130a $(\mathrm{P}<0.01$, Fig. 5B). In contrast, inhibiting the expression of miR-130a in MG63 cells resulted in significantly increased level of PTEN mRNA $(\mathrm{P}<0.01$, Fig. 5C) and protein $(\mathrm{P}<0.01$, Fig. 5D). Taken together, these data indicate the PTEN is a direct downstream target of miR$130 \mathrm{a}$, and miR-130a could regulate the expression of PTEN by interacting with the 3'-UTR of PTEN.

miR-130a promotes the metastasis and EMT of osteosarcoma cells by inhibiting PTEN. To further clarify whether PTEN can serve as the functional mediator of miR-130a in osteosarcoma cells, plasmids containing PTEN expressing vector and PTEN shRNA were transfected into HOS58 cells with overexpressed miR-130a (HOS58-miR-130a cells) and MG63 cells with downregulated miR-130a (MG63-anti-miR-130a cells), respectively. Functionally, overexpression of PTEN partly abrogated the promoting effects of miR-130a on the migration $(\mathrm{P}<0.05$, upper part of Fig. 6A) and invasion $(\mathrm{P}<0.05$, lower part of Fig. 6A) of HOS58 cells. Inhibiting PTEN expression in MG63-anti-miR-130a cells partly reversed the inhibitory effects of miR-130a on the migration $(\mathrm{P}<0.05$, upper part of Fig. 6B) and invasion $(\mathrm{P}<0.05$, upper part of Fig. 6B) of MG63 cells. Moreover, the results of western blot showed that transfection of PTEN expressing vector in HOS58-miR-130a cells resulted in significant increase of PTEN ( $\mathrm{P}<0.01$, Fig. 6C), and led to upregulation of E-cadherin $(\mathrm{P}<0.05$, Fig. 6C) and 

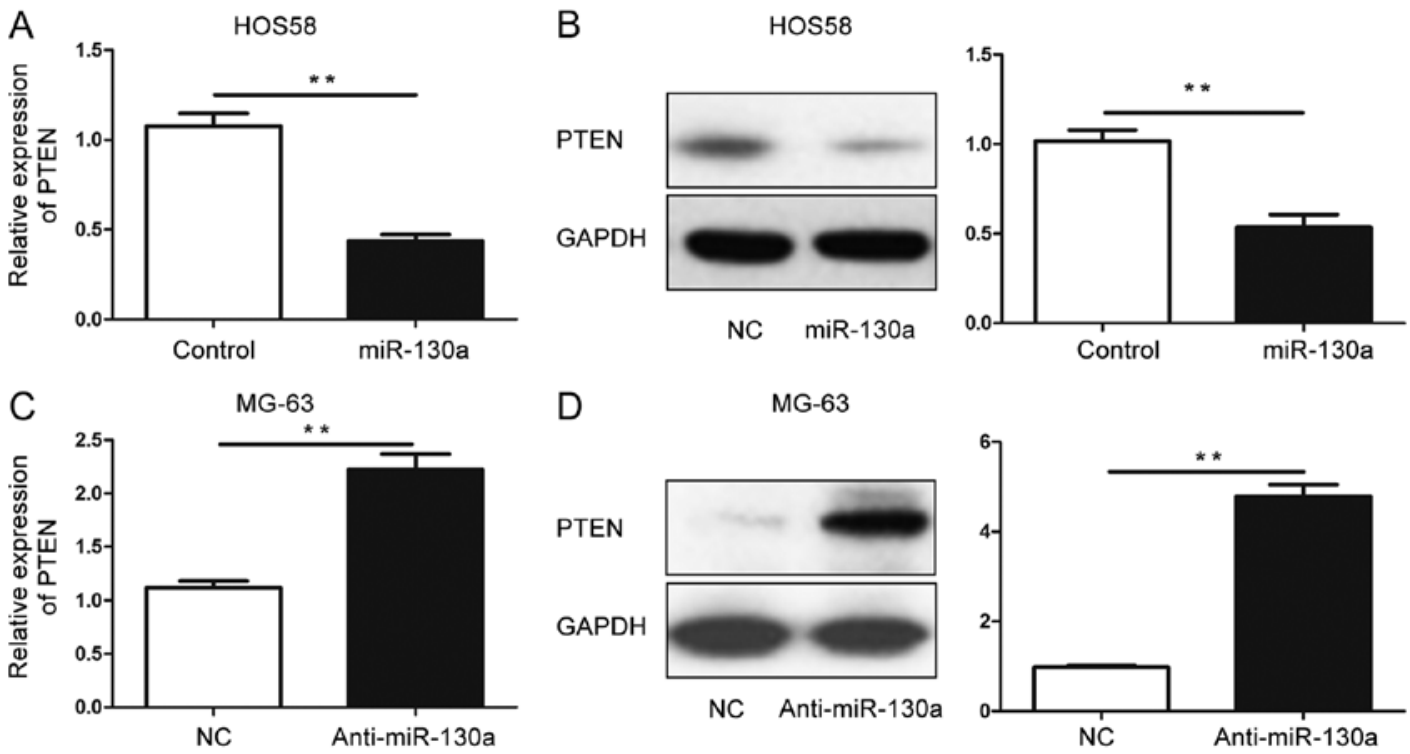

D

MG-63
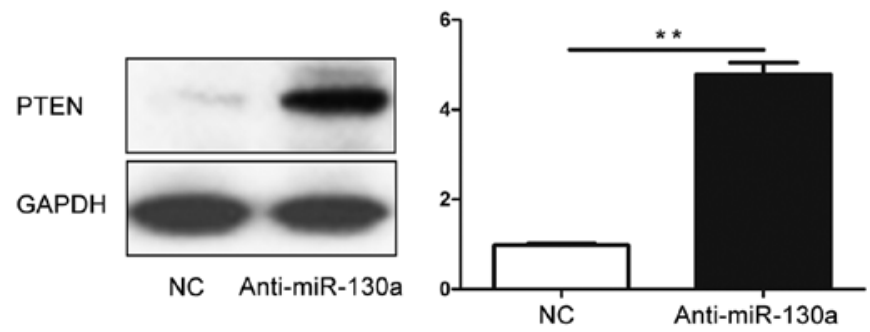

Figure 5. miR-130a regulates the expression of PTEN in osteosarcoma cells. Overexpression of miR-130a in HOS58 cells significantly inhibited the level of PTEN (A) mRNA and (B) protein. miR-130a and its predicted binding sequence in the 3'-UTR of PTEN. ${ }^{* *} \mathrm{P}<0.01$. Inhibition of miR-130a in MG63 cells significantly increased the level of PTEN (C) mRNA and (D) protein. ${ }^{* *} \mathrm{P}<0.01$.

A

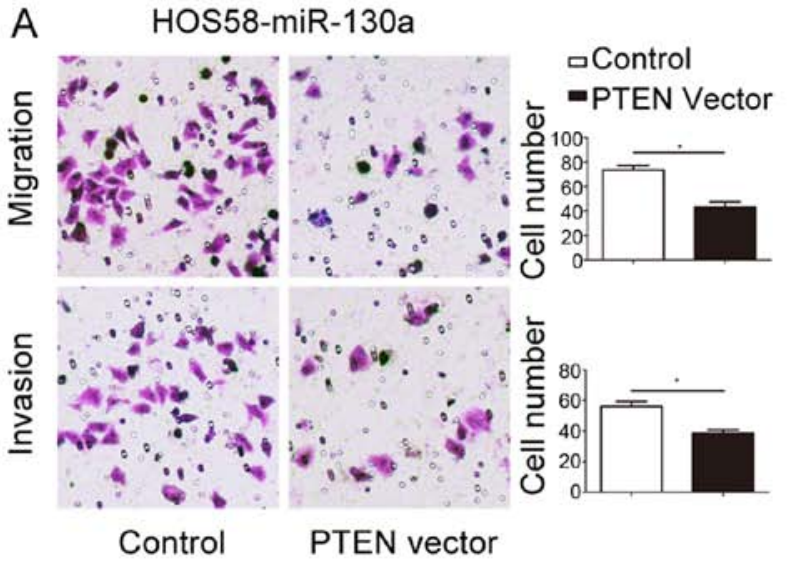

C

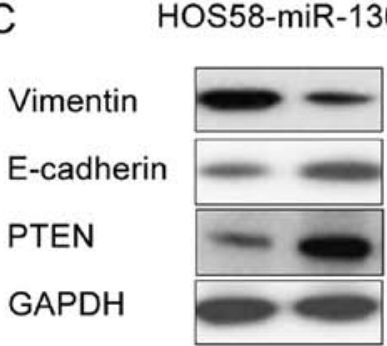

- Control

- PTEN Vector

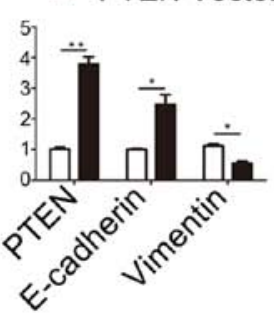

B MG63-anti-miR-130a

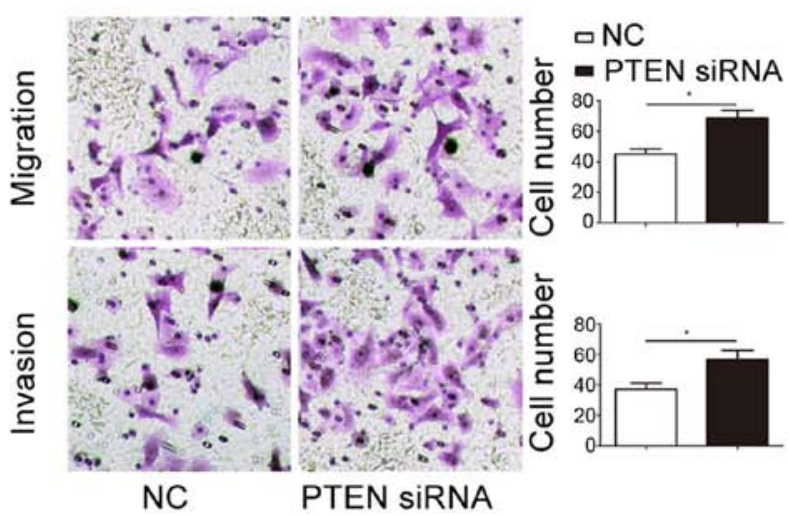

D MG63-anti-miR-130a

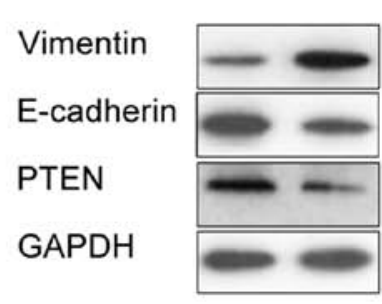

口 NC

- PTEN SIRNA

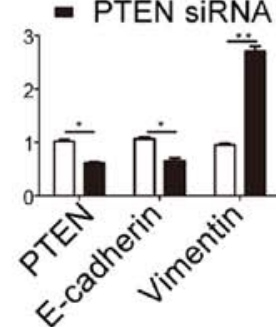

Figure 6. miR-130a exerts the promoting effects on the migration, invasion and EMT of osteosarcoma cells by suppressing PTEN. (A) Restoration of PTEN in HOS58-miR-130a cells abrogated the promoting effect of miR-130a mimics on cell migration and invasion. $\mathrm{n}=3$ repeats with similar results; ${ }^{*} \mathrm{P}<0.05$ (B) Inhibition of PTEN in MG63-anti-miR-130a cells reversed the inhibitory effect of miR-130a inhibitor on cell migration and invasion. n=3 repeats with similar results; ${ }^{*} \mathrm{P}<0.05$. (C) Western blot analysis of the expression of E-cadherin and Vimentin in HOS58-miR-130a cells transfected with PTEN vector and control vector; $\mathrm{n}=6 ;{ }^{*} \mathrm{P}<0.05,{ }^{* *} \mathrm{P}<0.01$. (D) Western blot analysis of the expression of E-cadherin and Vimentin in MG63-anti-miR-130a cells transfected with PTEN shRNA and negative control vector; $\mathrm{n}=6 ;{ }^{*} \mathrm{P}<0.05,{ }^{* *} \mathrm{P}<0.01$.

downregulation of Vimentin $(\mathrm{P}<0.05$, Fig. 6C). Transfection of PTEN shRNA in MG63-anti-miR-130a cells significantly inhibited the expression of PTEN (P<0.05, Fig. 6D), and resulted in the downregulation of E-cadherin $(\mathrm{P}<0.05$, Fig. 6D) and increase of Vimentin $(\mathrm{P}<0.01$, Fig. 6D).

\section{Discussion}

Metastasis is the culprit of the poor prognosis of osteosarcoma patients, and few effective treatments are available at present for these patients with metastasis (22). Metastasis of 
osteosarcoma cells is a complex process in which numerous molecules and signaling pathways are involved (23). Among them, microRNAs have been found to exert significant influence on this process (24). Identifying metastasis-related microRNAs can potentially help clinicians to find novel biomarkers and effective therapeutic targets for osteosarcoma patients.

miR-130a, which was recently identified as a cancerrelated microRNA, has been found to be abnormally expressed in various human malignancies, including gastric cancer $(8,9)$, esophageal cancer $(10)$, basal cell carcinoma (11), hepatocellular carcinoma $(12,13)$, prostate carcinoma $(14,15)$ and breast cancer $(16,17)$. However, the results of these studies showed miR-130a could play either oncogenic roles or tumor suppressive roles in different human cancers. In this study, we demonstrated for the first time that miR-130a was significantly overexpressed in osteosarcoma tissues and cell lines. Patients with metastasis had significantly higher expression level of miR-130a than those without metastasis. Furthermore, correlation analysis showed that increased level of miR-130a was closely associated with adverse clinical features (metastasis and advanced TNM stage) and poor prognosis (reduced OS and DFS) of osteosarcoma patients. These data indicate miR-130a plays oncogenic role in osteosarcoma and can probably contribute to the metastasis of osteosarcoma.

Functionally, miR-130a was found to promote the proliferation and angiogenesis of gastric cancer cells by targeting RUNX3 (9). miR-130a was confirmed to inhibit cell proliferation, invasion and migration of breast cancer cells by targeting the RAB5A (16). Moreover, a study of prostate cancer suggested that miR-130a was involved in the paclitaxel-resistance of prostate cancer cells (14). These studies show that the exact functional role of miR-130a in human cancers seems to be cancer-type specific. In this present study, the results of transwell assays showed that overexpression of miR-130a promoted the migration and invasion of HOS58 cells, while suppression of miR-130a attenuated the metastatic behavior of MG63 cells. These results indicate that miR-130a can promote the metastasis behavior of osteosarcoma cells. Furthermore, we confirmed that miR-130a could promote the EMT of osteosarcoma cells by examining the expression of EMT markers after overexpression or suppression of miR-130a. These data suggest that miR-130a can probably promote the metastasis by regulating the EMT process of osteosarcoma cells.

Phosphatase and tensin homolog (PTEN) is a widely accepted tumor suppressor (25), and has been found to regulate the proliferation, apoptosis and invasive behavior of osteosarcoma cells (26-28). Previous studies showed that PTEN was downstream target of miR-23 (29), miR-93 (30), miR-196a (31) and miR-214 (32) in osteosarcoma cells. In this study, we confirmed that PTEN was a downstream target of miR-130a. First, predicted binding sequences of miR-130a were found in the 3'-UTR of PTEN based on the data from two publicly available databases. Second, altering miR-130a level in osteosarcoma cells significantly influenced the luciferase activity of wt 3'-UTR of PTEN, but had no influence on that of mt 3'-UTR of PTEN, suggesting miR-130a could interact with the 3'-UTR of PTEN. Third, overexpression of miR-130a in HOS58 cells resulted in decreased, while suppression of miR-130a in MG63 led to increased, expression level of PTEN mRNA and protein, thus indicating that miR-130a can regulate the expression of PTEN by binding to the 3'-UTR region of PTEN.

Furthermore, we performed rescue experiments in HOS58miR-130a cells and MG63-anti-miR-130a cells to confirm that PTEN was not only a downstream target of miR-130a, but also a functional mediator of miR-130a in osteosarcoma cells. We found that restoring the expression of PTEN abrogated the promoting effects of miR-130a overexpression on metastatic behavior and EMT of HOS58 cells, while inhibiting the expression of PTEN reversed the inhibitory effects of miR130a knockdown on metastatic behaviors and EMT of MG63 cells. These data suggest that miR-130a exerts its functional effects on osteosarcoma cells by modulating the expression of PTEN.

In conclusion, this study confirms for the first time that miR-130a expression is significantly increased in osteosarcoma tissues and cells. The increased expression of miR-130a is associated with adverse prognostic features of osteosarcoma patients. miR-130a was identified as a valuable biomarker for the prognosis of osteosarcoma patients. Functionally, miR-130a can promote the migration, invasion and EMT of osteosarcoma cells. Mechanistically, this study demonstrates that PTEN is a downstream target of miR-130a in osteosarcoma and miR-130a exerts its functional significance on osteosarcoma cells by suppressing PTEN.

\section{Acknowledgements}

This study was supported by Scientific Research Foundation of Henan (no. 092102310090).

\section{References}

1. Torre LA, Bray F, Siegel RL, Ferlay J, Lortet-Tieulent J and Jemal A: Global cancer statistics, 2012. CA Cancer J Clin 65: 87-108, 2015.

2. Heymann D and Rédini F: Targeted therapies for bone sarcomas. Bonekey Rep 2: 378, 2013.

3. Yarber JL and Agulnik M: Targeted therapies in bone sarcomas: Current approach and future directions. Expert Opin Investig Drugs 20: 973-979, 2011.

4. Yates LA, Norbury CJ and Gilbert RJ: The long and short of microRNA. Cell 153: 516-519, 2013.

5. Rosa A and Brivanlou AH: MicroRNAs in early vertebrate development. Cell Cycle 8: 3513-3520, 2009.

6. Calin GA and Croce CM: MicroRNA signatures in human cancers. Nat Rev Cancer 6: 857-866, 2006.

7. Jansson MD and Lund AH: MicroRNA and cancer. Mol Oncol 6: 590-610, 2012.

8. Jiang H, Yu W-W, Wang L-L and Peng Y: miR-130a acts as a potential diagnostic biomarker and promotes gastric cancer migration, invasion and proliferation by targeting RUNX3. Oncol Rep 34: 1153-1161, 2015.

9. Lee SH, Jung YD, Choi YS and Lee YM: Targeting of RUNX3 by miR-130a and miR-495 cooperatively increases cell proliferation and tumor angiogenesis in gastric cancer cells. Oncotarget 6: 33269-33278, 2015.

10. Liu SG, Qin XG, Zhao BS, Qi B, Yao WJ, Wang TY, Li HC and $\mathrm{Wu} \mathrm{XN}$ : Differential expression of miRNAs in esophageal cancer tissue. Oncol Lett 5: 1639-1642, 2013.

11. Sand M, Skrygan M, Sand D, Georgas D, Hahn SA, Gambichler T, Altmeyer P and Bechara FG: Expression of microRNAs in basal cell carcinoma. Br J Dermatol 167: 847-855, 2012.

12. Li B, Huang P, Qiu J, Liao Y, Hong J and Yuan Y: MicroRNA-130a is down-regulated in hepatocellular carcinoma and associates with poor prognosis. Med Oncol 31: 230, 2014. 
13. Yang J, Han S, Huang W, Chen T, Liu Y, Pan S and Li S: A metaanalysis of microRNA expression in liver cancer. PLoS One 9: e114533, 2014

14. Fujita Y, Kojima T, Kawakami K, Mizutani K, Kato T, Deguchi T and Ito M: miR-130a activates apoptotic signaling through activation of caspase- 8 in taxane-resistant prostate cancer cells. Prostate 75: 1568-1578, 2015.

15. Boll K, Reiche K, Kasack K, Mörbt N, Kretzschmar AK, Tomm JM, Verhaegh G, Schalken J, von Bergen M, Horn F, et al: MiR-130a, miR-203 and miR-205 jointly repress key oncogenic pathways and are downregulated in prostate carcinoma. Oncogene 32: 277-285, 2013.

16. Pan Y, Wang R, Zhang F, Chen Y, Lv Q, Long G and Yang K: MicroRNA-130a inhibits cell proliferation, invasion and migration in human breast cancer by targeting the RAB5A. Int J Clin Exp Pathol 8: 384-393, 2015.

17. Stückrath I, Rack B, Janni W, Jäger B, Pantel K and Schwarzenbach H: Aberrant plasma levels of circulating miR-16, miR-107, miR-130a and miR-146a are associated with lymph node metastasis and receptor status of breast cancer patients. Oncotarget 6: 13387-13401, 2015.

18. Tsai JH and Yang J: Epithelial-mesenchymal plasticity in carcinoma metastasis. Genes Dev 27: 2192-2206, 2013.

19. Wei SC, Fattet L and Yang J: The forces behind EMT and tumor metastasis. Cell Cycle 14: 2387-2388, 2015.

20. Heerboth S, Housman G, Leary M, Longacre M, Byler S, Lapinska K, Willbanks A and Sarkar S: EMT and tumor metastasis. Clin Transl Med 4: 6, 2015.

21. Xu M, Jin H, Xu CX, Sun B, Song ZG, Bi WZ and Wang Y: miR-382 inhibits osteosarcoma metastasis and relapse by targeting Y box-binding protein 1. Mol Ther 23: 89-98, 2015.

22. Benjamin RS: Osteosarcoma: Better treatment through better trial design. Lancet Oncol 16: 12-13, 2015.
23. Kansara M, Teng MW, Smyth MJ and Thomas DM: Translational biology of osteosarcoma. Nat Rev Cancer 14: 722-735, 2014.

24. Jones KB, Salah Z, Del Mare S, Galasso M, Gaudio E, Nuovo GJ, Lovat F, LeBlanc K, Palatini J, Randall RL, et al: miRNA signatures associate with pathogenesis and progression of osteosarcoma. Cancer Res 72: 1865-1877, 2012.

25. Parsons R and Simpson L: PTEN and cancer. In: Tumor Suppressor Genes. El-Deiry WS (ed). Springer, New York, pp147-166, 2003.

26. Nielsen-Preiss SM, Silva SR and Gillette JM: Role of PTEN and Akt in the regulation of growth and apoptosis in human osteoblastic cells. J Cell Biochem 90: 964-975, 2003.

27. Kun C, Zongsheng Y and Yong H: Effects of PTEN gene transfection on apoptosis of human osteosarcom cell line MG-63 [J]. Acta Universitatis Medicinalis Anhui 5: 012, 2007.

28. Hu Y, Xu S, Jin W, Yi Q and Wei W: Effect of the PTEN gene on adhesion, invasion and metastasis of osteosarcoma cells. Oncol Rep 32: 1741-1747, 2014.

29. Tian K, Di R and Wang L: MicroRNA-23a enhances migration and invasion through PTEN in osteosarcoma. Cancer Gene Ther 22: 351-359, 2015.

30. Kawano M, Tanaka K, Itonaga I, Ikeda S, Iwasaki T and Tsumura H: microRNA-93 promotes cell proliferation via targeting of PTEN in Osteosarcoma cells. J Exp Clin Cancer Res 34: 76, 2015.

31. Shang Y, Wang L-Q, Guo Q-Y and Shi T-L: MicroRNA-196a overexpression promotes cell proliferation and inhibits cell apoptosis through PTEN/Akt/FOXO1 pathway. Int J Clin Exp Pathol 8: 2461-2472, 2015.

32. Liu CJ, Yu KL, Liu GL and Tian DH: MiR-214 promotes osteosarcoma tumor growth and metastasis by decreasing the expression of PTEN. Mol Med Rep 12: 6261-6266, 2015. 